\title{
Aluminum Stabilized NbTi Conductor Test Coil Design, Fabrication, and Test Results
}

\author{
N. Andreev, G. Chlachidze, D. Evbota, V.S. Kashikhin, M. Lamm, A. Makarov, T. Nakamoto, T. \\ Ogitsu, K. Tanaka, M. Tartaglia, A. Yamamoto, M. Yoshida
}

\begin{abstract}
A new generation of precision muon conversion experiments is planned at both Fermilab and KEK. These experiments will depend upon a complex set of solenoid magnets for the production, momentum selection and transport of a muon beam to a stopping target, and for tracking detector momentum analysis of candidate conversion electrons from the target. Baseline designs for the production and detector solenoids use NbTi cable that is heavily stabilized by an extruded high RRR aluminum jacket. A U.S.-Japan research collaboration has begun whose goal is to advance the development of optimized AlNbTi conductors, gain experience with the technology of winding coils from this material, and test the conductor performance as modest length samples become available. For this purpose, a "conductor test" solenoid with three coils was designed and built at Fermilab. A sample of the RIKEN Al-NbTi conductor from KEK was wound into a "test" coil; this was sandwiched between two "field" coils wound from doubled SSC cable, to increase the peak field on the RIKEN test coil. All three solenoid coils were epoxy impregnated, and utilized aluminum outer bandage rings to apply preload to the coils when cold. The design and fabrication details, and results of the magnet quench performance tests are presented and discussed.
\end{abstract}

Index Terms-Aluminum Stabilizer, NbTi, Superconductor, Solenoid

\section{INTRODUCTION}

$\mathrm{R}$ ESEARCHERS in particle physics are planning experiments at Fermilab in the U.S. [1] and at J-PARC in Japan [2] to search with greatly increased sensitivity, four orders of magnitude beyond previous searches, for evidence of direct muon conversion to electrons. This "Mu2e" process is highly suppressed in the standard model (SM), but many theories beyond the SM predict new particles that could mediate such events at levels that would be detectable by these new experiments. To achieve such dramatic improvement will require the production and transport of an intense, low background muon beam to a stopping target, then the capture and highly precise momentum analysis of electrons.

Manuscript received 12 September 2011. This work was supported in part by Fermi Research Alliance, LLC, under contract No. DE-AC02-07CH11359 with the U.S. Department of Energy, and in part by the Japan-U.S. cooperative program in High Energy Physics.

N. Andreev, G. Chlachidze, D. Evbota, V.S.Kashikhin, M. Lamm, A. Makarov, and M. Tartaglia are with the Fermi National Accelerator Laboratory, Batavia IL 60510 USA (corresponding author phone: 630-8403890; fax: 630-840-8079; e-mail: tartaglia@fnal.gov).

T. Nakamoto, T. Ogitsu, K. Tanaka, A. Yamamoto, and M. Yoshida are with the KEK laboratory, Tsukuba, Japan (e-mail: ogitsu@ post.kek.jp).
Thus each experiment must rely heavily on the design and performance of a complex superconducting magnet system [3]. To create an intense muon beam requires efficient collection of low energy pions emitted from a production target located within a "capture" or "production" solenoid (CS in COMET, PS in Mu2e) which have a peak field of $5 \mathrm{~T}$. Among the many magnet challenges, one of the most fundamental is to develop a conductor of performance suitable to operate at this field in a high radiation environment.

Use of $\mathrm{Cu}-\mathrm{NbTi}$ superconductor (SC) that is heavily stabilized with an extruded aluminum jacket is preferred for the "production" and "detector" solenoids which will be cooled by conduction. Due to its light weight and high strength, transparency to radiation and reduction of neutron heat deposition, aluminum is favored over copper. Reduction of RRR due to cold irradiation can be recovered in aluminum with room temperature annealing, while copper requires higher temperature to fully recover.

Delivery of the solenoid magnets will determine the time scale for doing the experiments. Development and optimization of the conductor for these magnets is on the critical path for starting the solenoid fabrication. Thus a U.S.Japan collaboration formed to investigate the important conductor R\&D issues, and gain experience with the technologies needed to manufacture solenoid coils with this material and test the performance of modest length samples of the conductor after winding them into coils. The availability of a conductor sample from RIKEN superconducting ring cyclotron magnets [4] afforded an opportunity to begin this R\&D. The RIKEN cable is comprised of ten strands of 1.15 $\mathrm{mm}$ diameter $\mathrm{Cu}-\mathrm{NbTi}(\mathrm{Cu} / \mathrm{SC}=0.9)$ in a 2-layer Rutherford cable, co-extruded in the center of a pure aluminum stabilizer (RRR>500) with $8 \mathrm{~mm}$ x $15 \mathrm{~mm}$ rectangular cross section.

\section{DESIGN AND FABRICATION}

A primary and reasonable goal of the $R \& D$ is to study the test conductor behavior at high field, to see how it performs in a high field of at least $5 \mathrm{~T}$. However, for the modest length of Al-NbTi available, about $120 \mathrm{~m}$, the self-field of the Test coil alone does not approach this. To increase the total field, the Test coil was positioned between, and in series with, two other field-generating ("Main" or "Field") coils having sufficient amp-turns to generate a $5 \mathrm{~T}$ field well below the expected critical surface of the Test coil. Cable left over from the SSC dipole program (SSC Inner cable) was chosen to wind the Field coils, and has quite similar measured critical current as 
the RIKEN cable. The Field coil windings utilized doubled SSC cable, with the keystone angles reversed to make a flat cross section for winding, so that the Test coil critical surface would limit quench performance.

A schematic view of the solenoid is shown in Fig. 1, and the fabrication details are documented in a production report [5]. The magnet outer diameter is constrained by the inner dimension of the cryogenic test dewar, the Fermilab Vertical Magnet Test Facility (VMTF). Two Kapton-insulated stainless steel strip heaters were positioned between the bobbin and the inner layer of the Test coil. As is planned for the Mu2e solenoids, the Test coil was wound from pre-insulated conductor in the "hard way bend" orientation, bending in the cable thicker direction. Specially shaped G10 blocks and fiberglass cloth were used to fill voids in the coil and facilitate cable tensioning at the vertical interlayer transitions. The Test coil consisted of 80 turns in 8 vertical by 10 horizontal layers.

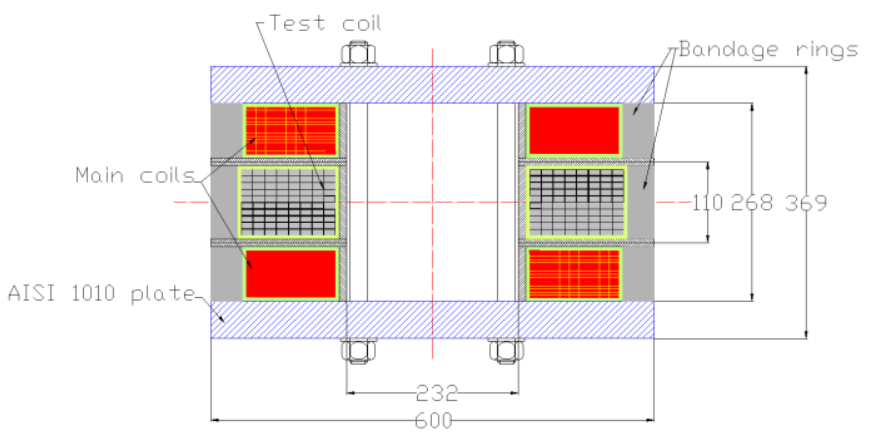

Fig. 1. Schematic view of conductor test solenoid, Mu2e_SOL_01.

The Field coil doubled cables were similarly pre-insulated and wound in "hard way bend", to eliminate flux differences between the doubled cables that may generate circulating currents. Each Field coil consisted of 160 turns in 10 vertical by 16 horizontal layers.

After winding around an insulated $316 \mathrm{~L}$ stainless steel bobbin, an outer $\mathrm{Al}$ bandage ring was used to provide each coil with pre-stress (by compression against the inner bobbin which shrinks less) during cool down to liquid helium (LHe) temperature. The solenoid mechanical design utilized epoxy vacuum impregnation of the individual coils, which were then bolted together between end plates of low carbon steel to provide end pre-load and help reduce the peak field difference between coils. Finite element analysis indicates the mechanical stresses are below the materials cryogenic maximum allowable ( $<400 \mathrm{MPa}$ in the end plates), and the peak coil Lorentz force is $38 \mathrm{kN}$ in the Main coils.

The leads for each coil were mechanically secured outside the coil package, and NbTi to NbTi soldered splices were made and heavily stabilized by blocks of copper. The long leads connecting to the test facility power leads were of doubled SSC cable that were mechanically secured to prevent motion and quenching in the large solenoid fringe field. Voltage taps for quench location were placed across each coil.

\section{TEST AND RESULtS}

\section{A. Test Overview}

A single thermal cycle cold test of the Mu2e_SOL_01 magnet was performed in October 2010 [6]. The magnet passed all warm and cold electrical tests, including a $1000 \mathrm{~V}$ to ground "hipot" insulation check at cold. During quench testing, the $\sim 19 \mathrm{mH}$ solenoid was protected using a $90 \mathrm{~m} \Omega$ dump resistor. Though $\mathrm{Mu} 2 \mathrm{e}$ magnets will be conductioncooled, quench training was studied in liquid helium at $4.58 \mathrm{~K}$ and $3.0 \mathrm{~K}$ temperatures, and ramp rate dependence of the quench current was mapped out. Measurements of the magnetic field profile on the solenoid axis were performed at $1 \mathrm{kA}$ and $3 \mathrm{kA}$ using a Hall probe within a warm bore tube. Thermal margin of the Test Coil conductor was studied by powering the strip heaters at fixed solenoid currents. Finally, the average RRR of the individual coils was measured while warming the magnet after the test.

\section{B. Magnetic Field}

Magnetic field measurements were made to confirm the predicted on-axis profile for the as-built solenoid geometry. A 3-axis Senis $10 \mathrm{~T}$ Hall probe was scanned along the solenoid axis with $\sim 1 \mathrm{~mm}$ position accuracy. Two independent calculations of the field were made, one with 2D and one with 3D geometry, using Opera. Fig. 2 shows the measured and calculated field strength profile $\mathrm{B}_{\text {mod }}$ (which is axial except for small transverse components due to probe tilt) at $3000 \mathrm{~A}$. This agreement provides confidence in the calculated peak field on the coils, which is needed to predict the maximum quench current.

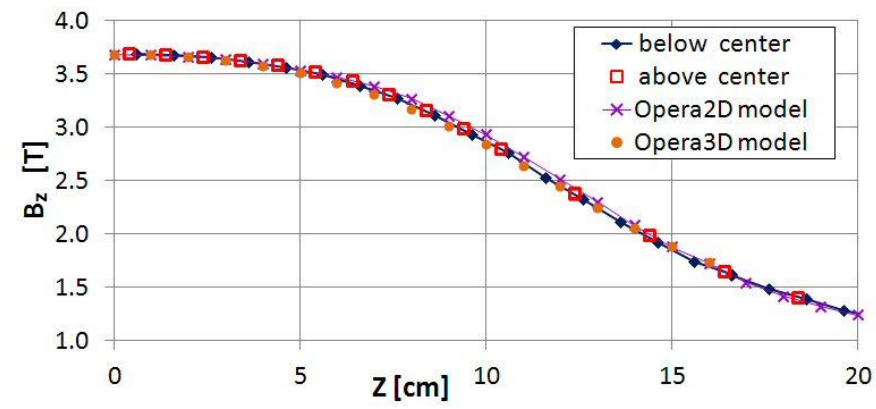

Fig. 2. Measured and calculated profiles of the axial magnetic field strength.

\section{Quench Performance}

The expected maximum quench current is illustrated in Fig. 3 by the intersection of the peak field load lines and the conductor critical surfaces. The figure shows the RIKEN data points at $4.3 \mathrm{~K}$, and the single SSC cable critical surface at the measured $4.2 \mathrm{~K}, 3.0 \mathrm{~K}$ and $4.58 \mathrm{~K}$ calculated values [7]. We therefore assume that RIKEN and single SSC cable performance are quite similar, and predict the Test coil should limit the solenoid quench current to $6(7) \mathrm{kA}$ at $4.6(3.0) \mathrm{K}$.

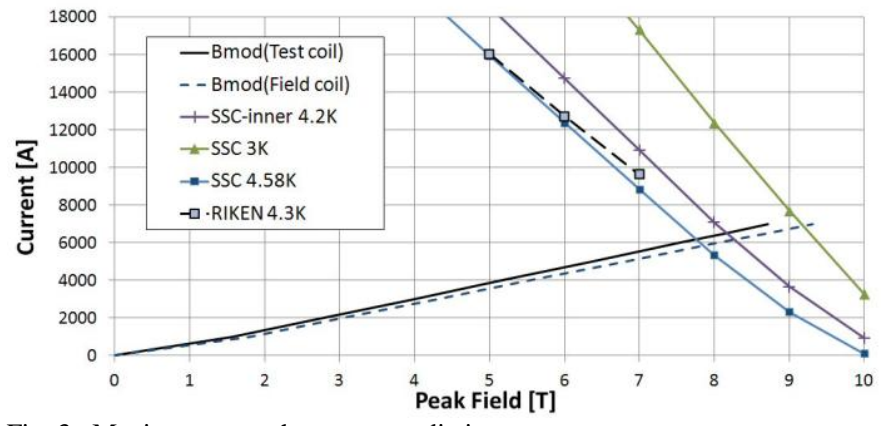

Fig. 3. Maximum quench current prediction. 
The quench training history is shown in Fig. 4. Initial quench training was slow and well below the expected maximum current. The initial ramp rate of $20 \mathrm{~A} / \mathrm{s}$ was reduced to $5 \mathrm{~A} / \mathrm{s}$, and although slow the training was steady. Quenches originated in all coils, and many were simultaneous in the Test and either Top or Bottom Field coil. Most quenches developed very quickly, within a few milliseconds; only five quenches developed in $>25 \mathrm{~ms}$, which occurred solely in the Top or Bottom coil. Multiple coil origins and slow training suggested origins of a mechanical nature, so temperature was reduced to $3.0 \mathrm{~K}$ to look for improvement in the training rate, which remained the same. Eventually the ramp rate limited the quench current, and improvement was possible only at very low rate, $1 \mathrm{~A} / \mathrm{s}$. The ramp rate dependence of quench current is shown in Fig. 5 with locations shown for the highest point at each rate.

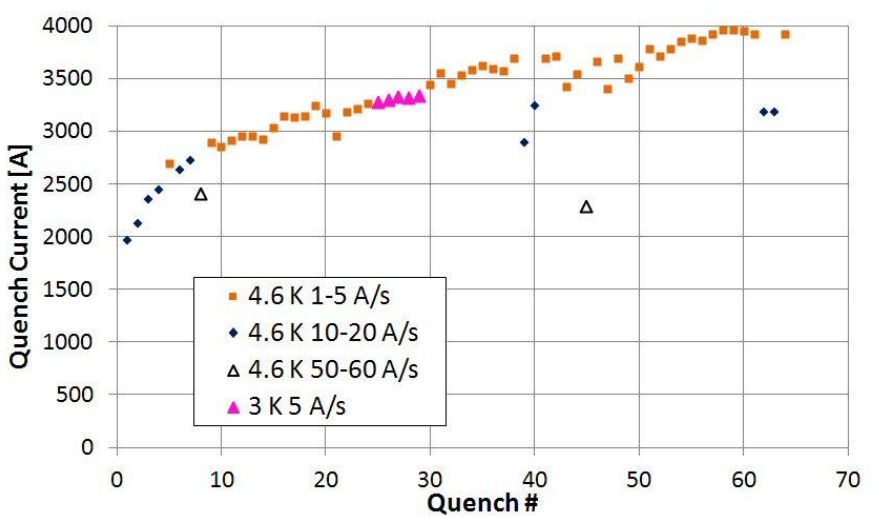

Fig. 4. Mu2e_SOL_01 quench training history.

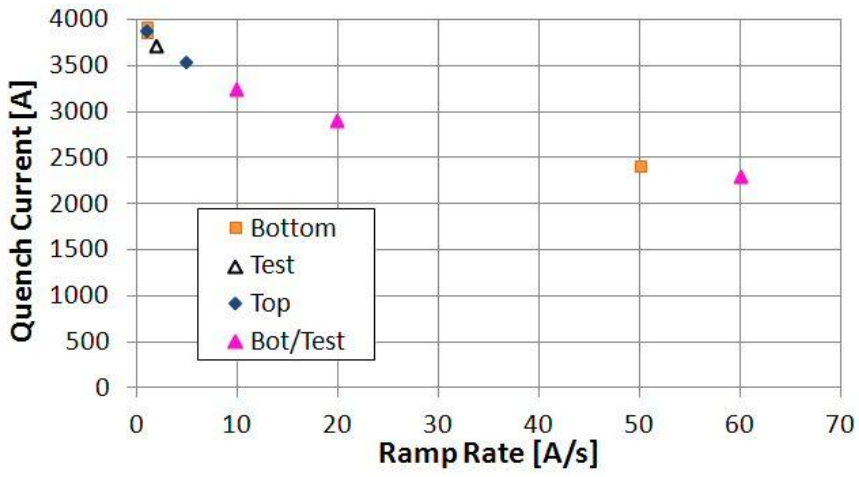

Fig. 5. Ramp rate dependence of quench current.

A frequency plot of quench locations is shown in Fig. 6. Quite apart from the expectation that the Test coil should limit performance, it is clear that Field coil quenches dominated despite the doubled cable design. That combined with the lack of training rate improvement at lower temperature, and multiple coil fast quenches, suggests severe mechanical limitation. The strong ramp rate dependence is likely due to heating from eddy currents in the aluminum bandage and perhaps more importantly - in the $15 \mathrm{~mm}$ wide stabilizer of the RIKEN conductor, which can contribute heat at the interface between the coils and near the peak field region. We hypothesize that this heat would raise the coil temperature and may result in local stress changes in the epoxy structure. Also, the inter-cable contact for the doubled cable is not well controlled, so there may be circulating current effects, and non-uniform current distribution in the Field coils. No attempt has been made to calculate such effects, however.

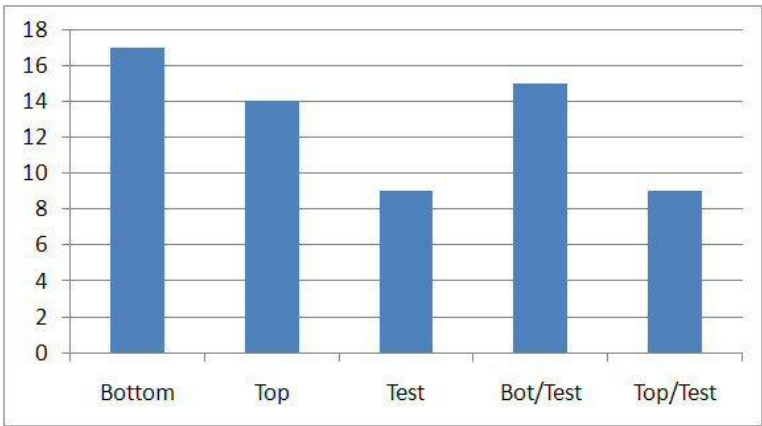

Fig. 6. Frequency plot of quench locations.

It is important to note that, despite the magnet quench limitations, at the highest current of $\sim 4 \mathrm{kA}$ the Test coil reached the desired peak field of $5 \mathrm{~T}$ on the coil (see Fig. 3) and the Test coil did not limit the solenoid performance.

\section{Thermal Margin Study}

Being limited by the achievable quench current, another approach was taken to reach the Test coil conductor critical surface. With the solenoid operating at fixed current, the strip heaters were energized to elevate the temperature of the Test coil, by slowly increasing the heater current until a quench was induced. Fig. 7 shows the total heater power required to induce a quench as a function of the solenoid current; the simulation shown is discussed later. Three tests were made at the $3000 \mathrm{~A}$ setting, to gauge the reproducibility of the measurement, and a single test at each of the other currents.

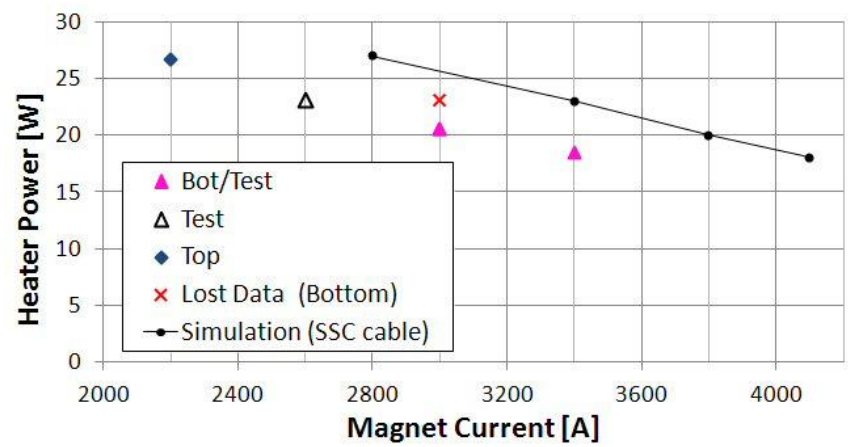

Fig. 7. Strip heater power needed to induce a quench versus solenoid current, showing actual quench locations and thermal model calculated critical surface.

The quench development in these events was very interesting: all events above 2400 A show a slow voltage growing in the Test coil only prior to the quench, which is evidence of a growing normal zone and indicates an approach to the critical surface. However, in most cases the quenches originated elsewhere (though voltage segment data were lost in the $3^{\text {rd }} 3000 \mathrm{~A}$ test, independently logged half coil signals indicate a Bottom coil origin there). Furthermore, at $2200 \mathrm{~A}$ the voltage signals (Fig. 8) suggest a large mechanical disturbance at the start of the Top coil quench, followed by another during the quench. Thus, a possible scenario is that the local temperature increase results in sudden stress relief events that actually induce the quenches. 


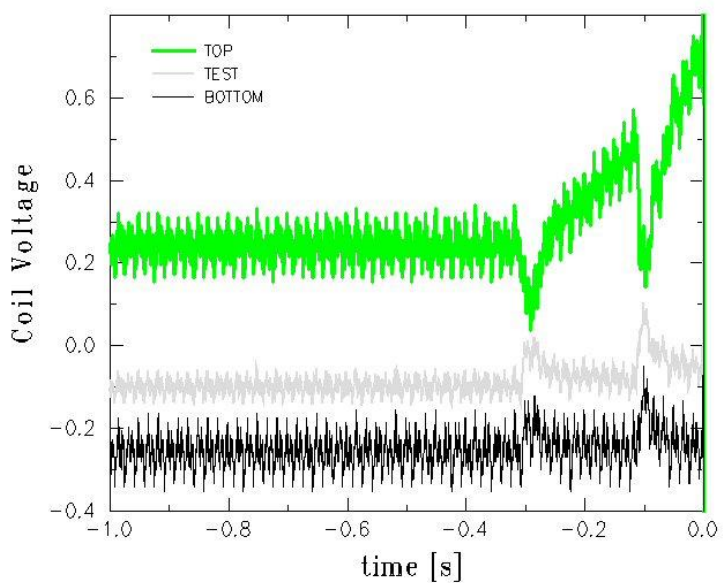

Fig. 8. Voltage Tap signals showing Top coil quench event during 2200 A thermal margin test (traces have been offset vertically for clarity).

To interpret the results of Fig. 7 and compare them to the expected (parametrized) critical surface requires a calculation of the coil temperature as a function of the heat flux. For this purpose a 2D finite element thermal model was constructed in ANSYS Workbench with the detailed structure of the Test coil cross section, from the helium bath at $4.6 \mathrm{~K}$, a convective layer at the surface, bobbin, insulation layers, heaters, coil and bandage. The coil is simply modeled as a monolithic block of aluminum. A layer of G10 insulation separates the Test and Field coils. Fig. 9 shows an example of the temperature profile from the simulation, for 27 Watt heat flux emitted by two displaced strip heaters inside the Test coil winding.

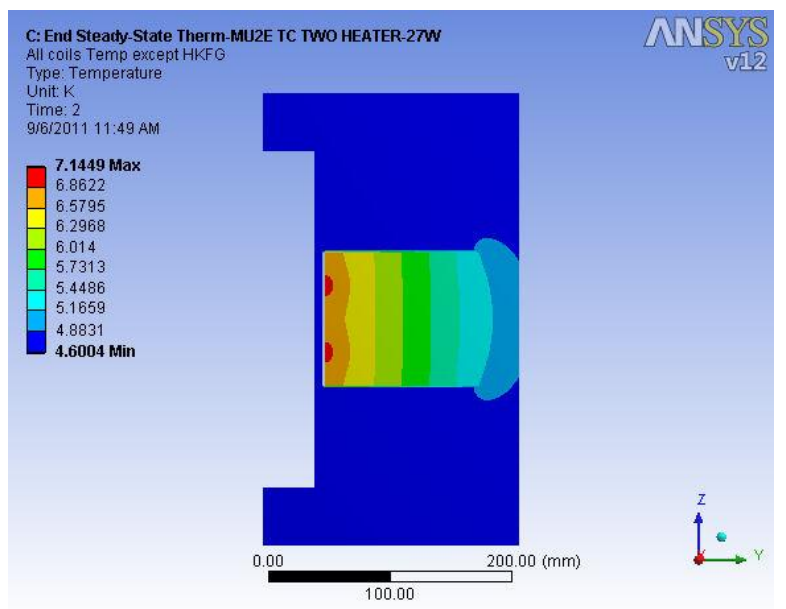

Fig. 9. 2D thermal model temperature profile at 27 Watts dissipated equally in two heaters inside the Test coil.

Table I shows the predicted coil temperature as a function of the heat flux, the expected critical current (for the SSC cable using [7] to parametrize the temperature dependence), and the magnet currents which were shown also in Fig. 7. Sensitivity to the thermal properties of the materials is still under study, especially the uncertainty on the thermal conductivity of the RIKEN aluminum stabilizer. The modeled Test coil substructure will also be improved. The modest agreement in Fig. 7 suggests this could be a promising alternative way to investigate the conductor Ic and stabilizer thermal properties in the future.
TABLE I THERMAL MODEL RESULTS

\begin{tabular}{cccc}
\hline $\begin{array}{c}\text { Heater } \\
\text { Power [W] }\end{array}$ & $\begin{array}{c}\text { Test Coil } \\
\text { Temperature [K] }\end{array}$ & Predicted Ic [A] & $\begin{array}{c}\text { Actual Quench } \\
\text { Current [A] }\end{array}$ \\
\hline 18 & 6.30 & 4100 & 3400 \\
20 & 6.48 & 3800 & 3000 \\
23 & 6.77 & 3400 & 2600 \\
27 & 7.15 & 2800 & 2200 \\
\hline \hline
\end{tabular}

\section{E. RRR Measurement}

The RRR determination was made as the ratio of coil resistance at room temperature to that just after superconductor transition to normal during the warm up, at about $9 \mathrm{~K}$. The Test coil value 857 is in good agreement with the RIKEN specification sheet value of 912, and is perhaps slightly lower as a result of the coil winding process. The Field coils had reasonable RRR values of 115 (Bottom) and 117 (Top).

\section{CONCLUSION}

Motivated by the solenoid magnet challenges in planned muon conversion search experiments, a test solenoid was built to gain experience with fabrication of coils made from aluminum-stabilized NbTi Rutherford cable. A Test coil made from RIKEN Al-NbTi conductor was sandwiched between two other coils of doubled SSC cable to generate background field. All coils were epoxy impregnated and operated in series.

Quench performance tests showed mostly multiple coil quench origins, and the magnet was clearly limited by mechanical issues rather than conductor critical current. The Test coil reached the desired $5 \mathrm{~T}$ field level, but strong ramp rate dependence did not allow reaching higher fields to directly find the actual conductor critical current. The Test coil temperature was raised with heaters to study thermal margin to the critical surface, and these quench current results can be reasonably interpreted using a detailed thermal model.

\section{ACKNOWLEDGMENT}

The authors express appreciation to the RIKEN Nishina Center for Accelerator-Based Science [8] for the sample of aluminum stabilized superconductor used for this study.

\section{REFERENCES}

[1] http://mu2e.fnal.gov/

[2] http://comet.phys.sci.osakau.ac.jp:8080/comet/internal/publications/comet-cdr-v1.0.pdf

[3] M. Lamm, et al., "Solenoid Magnet System for the Fermilab Mu2e Experiment," Submitted for publication to this conference, 1AO-6.

[4] A. Goto, et al., "Sector Magnets for the RIKEN Superconducting Ring Cyclotron," IEEE Trans. Appl. Supercond., Vol 14, No. 2, pp 30003005, June 2004.

[5] D. Evbota, V.S. Kashikhin, A. Makarov, "Aluminum Stabilized NbTi Test Coil Assembly Production Report," TD-10-011, FNAL, Nov. 2010.

[6] N. Andreev, et al., "Mu2e_SOL_01 Magnet Test Summary," TD-11013, FNAL, Sept. 2011.

[7] L. Bottura, "A practical fit for the critical surface of NbTi," IEEE Trans. Appl. Supercon., Vol. 10, No. 1, pp.1054-1057, 2000.

[8] http://www.rarf.riken.jp/Eng/index.html 